\title{
Two-loop soft anomalous dimensions and NNLL resummation for heavy quark production
}

\author{
Nikolaos Kidonakis \\ Kennesaw State University, Kennesaw, GA 30144, USA
}

\begin{abstract}
I present results for two-loop soft anomalous dimensions for heavy quark production which control soft-gluon resummation at next-to-next-to-leading-logarithm (NNLL) accuracy. I derive an explicit expression for the exact result and study it numerically for top quark production via $e^{+} e^{-} \rightarrow t \bar{t}$, and I construct a surprisingly simple but very accurate approximation. I show that the two-loop soft anomalous dimensions with massive quarks display a simple proportionality relation to the one-loop result only in the limit of vanishing quark mass. I also discuss the extension of the calculation to single top and top pair production in hadron colliders.
\end{abstract}


The calculation of higher-order corrections to heavy quark cross sections and differential distributions is needed for increasing the accuracy of the theoretical predictions. Given the importance of the top quark [1] to electroweak and Higgs physics, the top quark cross section at the Tevatron and the LHC, both in top pair [2] and single-top [3] production modes, as well as at a future $e^{+} e^{-}$collider needs to be calculated with the highest possible precision. Bottom and charm quark cross sections are also important in understanding QCD and calculating backgrounds to new physics.

Soft-gluon emission is an important contributor to higher-order corrections, particularly near partonic threshold. The soft-gluon corrections can be formally resummed to all orders in perturbation theory. The resummation arises from the factorization of the cross section into a hard-scattering function $H$ and a soft function $S$ that describes noncollinear soft-gluon emission in the process [4]. The evolution of the soft function is controlled by a process-dependent soft anomalous dimension $\Gamma_{S}$. The resummed cross section can be written in moment space as $\sigma^{\text {res }}(N)=\exp \left[E_{i}(N, \mu)\right] H(\mu) S(N) \exp \left[\int_{m}^{m / N}(d \mu / \mu) 2 \Gamma_{S}(\mu)\right]$, with $\mu$ the factorization scale, $m$ the heavy quark mass, and $N$ the moment variable which is conjugate to a kinematical variable measuring distance from partonic threshold. The exponents $E_{i}$ resum collinear and soft radiation from any massless partons in the scattering. The logarithms of $N$ exponentiate. At leading-logarithm accuracy we determine the coefficients of the highest power of $\ln N$ at each order in the strong coupling $\alpha_{s}$, at next-to-leading logarithm (NLL) accuracy we also determine the second highest power of $\ln N$, etc.

For heavy quark pair, such as $t \bar{t}$, hadroproduction the soft anomalous dimensions are matrices in color space due to the complicated color structure of the scattering processes, and were calculated at one loop for both $q \bar{q} \rightarrow t \bar{t}$ and $g g \rightarrow t \bar{t}$ channels in Ref. [4], thus allowing resummation at next-to-leading logarithm. From the calculation in [4] one can also derive the corresponding result for heavy quark production at $e^{+} e^{-}$machines, a simple function related to the cusp anomalous dimension of Ref. [5]. For single top production at hadron colliders the one-loop soft anomalous dimensions were presented for the $t, s$, and $t W$ channels in Ref. [6]. To increase the accuracy of the resummation to next-to-next-to-leading logarithms (NNLL) for all these processes one needs to derive the soft anomalous dimensions at two loops.

The calculations of soft anomalous dimensions involve diagrams with eikonal (Wilson) lines representing the heavy quarks. The eikonal approximation is valid for describing the emission of soft gluons from particles in the hard scattering and leads to a modified form of the Feynman rules and thus of diagram calculations (see e.g. [4-9]). When the gluon momentum goes to zero, the quark-gluon vertex reduces to $g_{s} T_{F}^{c} v^{\mu} / v \cdot k$, with $g_{s}$ the strong coupling, $v$ a dimensionless velocity vector, $k$ the gluon momentum, and $T_{F}^{c}$ the generators of $\mathrm{SU}(3)$ in the fundamental representation. Below we calculate explicitly the soft anomalous dimension through two loops for the process $e^{+} e^{-} \rightarrow t \bar{t}$, study its properties in detail and then discuss the extension to processes with more complicated color structure, i.e. single top and top pair hadroproduction.

The eikonal diagrams are calculated in dimensional regularization with $n=4-\epsilon$ and in Feynman gauge in momentum space. The one-loop soft anomalous dimension, $\Gamma_{S}^{(1)}$, can be read off the coefficient of the ultraviolet (UV) pole of the one-loop diagrams in Fig. 1. Writing 


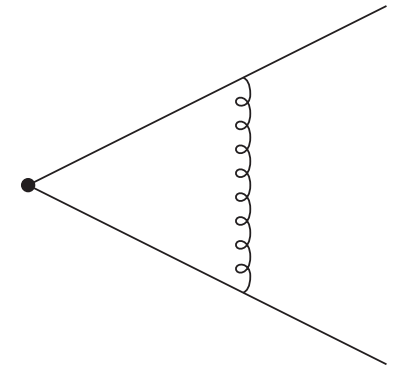

(a)

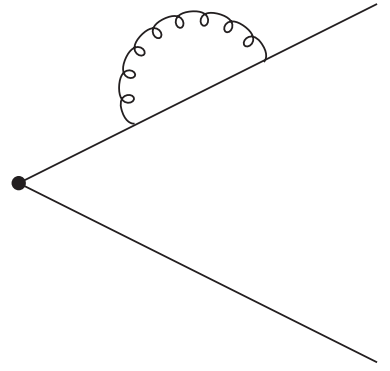

(b)

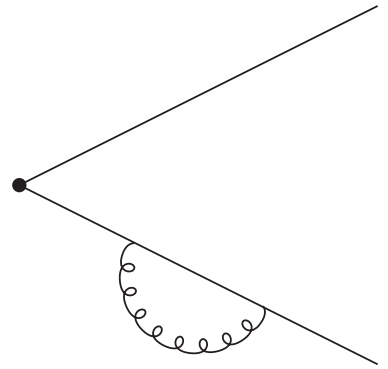

)

Figure 1: One-loop diagrams with heavy-quark eikonal lines.

$\Gamma_{S}=\left(\alpha_{s} / \pi\right) \Gamma_{S}^{(1)}+\left(\alpha_{s} / \pi\right)^{2} \Gamma_{S}^{(2)}+\cdots$, we find the one-loop expression

$$
\Gamma_{S}^{(1)}=C_{F}\left[-\frac{\left(1+\beta^{2}\right)}{2 \beta} \ln \left(\frac{1-\beta}{1+\beta}\right)-1\right]
$$

with $C_{F}=4 / 3, \beta=\sqrt{1-4 m^{2} / s}$, and $s=\left(p_{e^{+}}+p_{e^{-}}\right)^{2}$.

We now continue with the two-loop diagrams. A few results for some of these diagrams have appeared in [8, 9, 10]. In Fig. 2 we show graphs with vertex corrections and in Fig. 3 graphs with heavy-quark self-energy corrections. There are three more graphs, not shown, with vanishing contributions: two graphs with gluon loops involving a four-gluon vertex, and one graph involving a three-gluon vertex with all three gluons attaching to a single eikonal line. The calculations are challenging due to the heavy quark mass and involve multiple complicated integrals and delicate separations of infrared and ultraviolet poles (which by construction of the soft function are opposites of each other [4]). The analytical structure involves logarithms and polylogarithms. It is understood in the results below that for each diagram we include the appropriate one-loop counterterms for the divergent subdiagrams (but note that graph 2(b) does not have a divergent subdiagram). Typically there are large cancellations of terms between the individual diagrams and their respective counterterms.

We now present the UV poles of the kinematic terms for the two-loop diagrams, which later will have to be combined with color and symmetry factors, and an overall factor $\alpha_{s}^{2} / \pi^{2}$. The sum of the UV poles of diagrams $2(\mathrm{a})$ and $2(\mathrm{~b})$ is minus one-half the square of the UV pole of the one-loop diagram of Fig. 1(a): $I_{2 a}+I_{2 b}=\left[\left(1+\beta^{2}\right)^{2} /\left(8 \beta^{2}\right)\right]\left(-1 / \epsilon^{2}\right) \ln ^{2}[(1-\beta) /(1+\beta)]$. The UV poles of the crossed diagram, Fig. 2(b), are

$I_{2 b}=\frac{\left(1+\beta^{2}\right)^{2}}{8 \beta^{2}} \frac{1}{\epsilon}\left\{-\ln \left(\frac{1-\beta}{1+\beta}\right)\left[\operatorname{Li}_{2}\left(\frac{(1-\beta)^{2}}{(1+\beta)^{2}}\right)+\zeta_{2}\right]-\frac{1}{3} \ln ^{3}\left(\frac{1-\beta}{1+\beta}\right)+\operatorname{Li}_{3}\left(\frac{(1-\beta)^{2}}{(1+\beta)^{2}}\right)-\zeta_{3}\right\}$.

The diagram in Fig. 2(c) represents corrections from quark, gluon, and ghost loops. The quark-loop contribution is $I_{2 c q}=\left[n_{f}\left(1+\beta^{2}\right) /(6 \beta)\right]\left[1 / \epsilon^{2}-5 /(6 \epsilon)\right] \ln [(1-\beta) /(1+\beta)]$ with $n_{f}$ the number of light quark flavors, while the gluon plus ghost contribution is $I_{2 c g}=(5 / 24)[(1+$ 


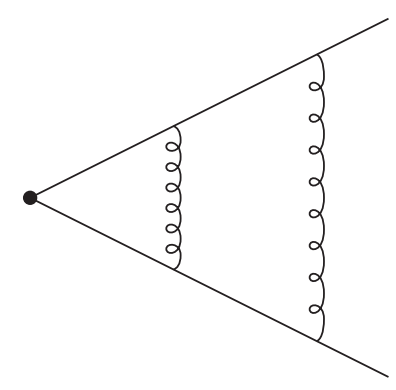

(a)

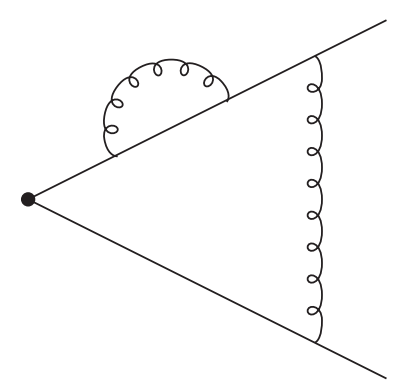

(d)

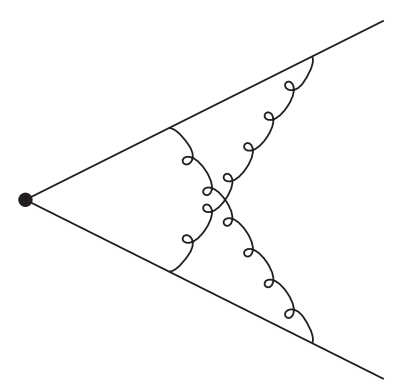

(b)

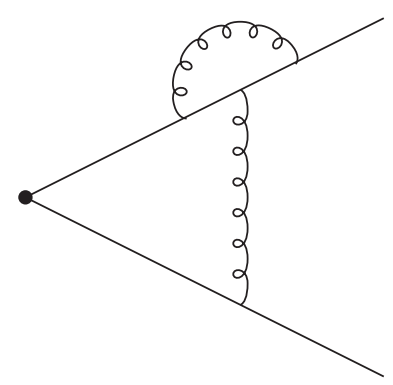

(e)

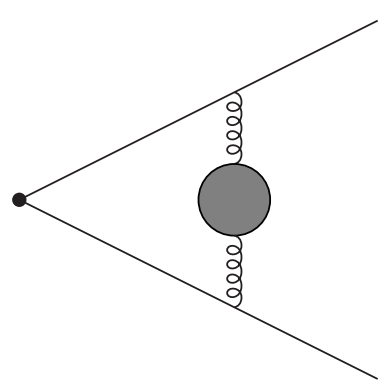

(c)

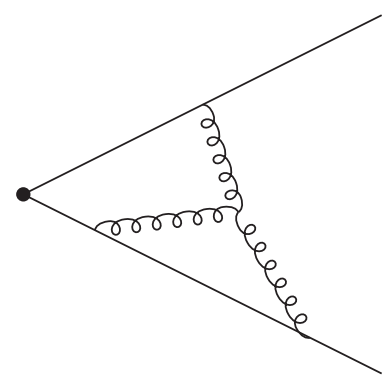

(f)

Figure 2: Two-loop vertex diagrams with heavy-quark eikonal lines.

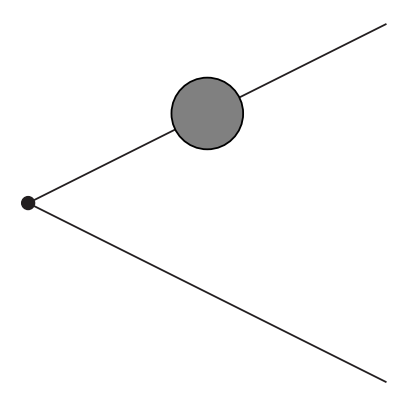

(a)

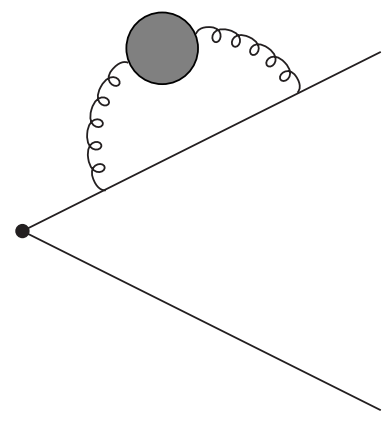

(b)

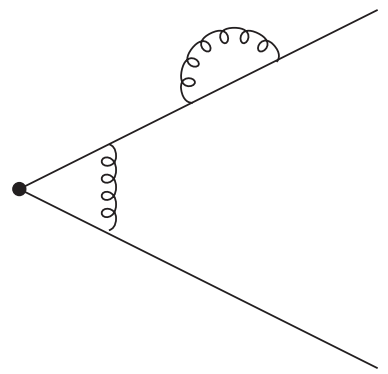

(c)

Figure 3: Two-loop heavy-quark self-energy diagrams with eikonal lines. 
$\left.\left.\beta^{2}\right) / \beta\right]\left[1 / \epsilon^{2}-31 /(30 \epsilon)\right] \ln [(1-\beta) /(1+\beta)]$. Diagram $2(\mathrm{~d})$ is given by

$$
\begin{aligned}
I_{2 d}= & \frac{\left(1+\beta^{2}\right)}{4 \beta}\left\{-\frac{1}{\epsilon^{2}} \ln \left(\frac{1-\beta}{1+\beta}\right)+\frac{1}{\epsilon}\left[\frac{1}{2} \ln ^{2}\left(\frac{1-\beta}{1+\beta}\right)+\ln \left(\frac{1-\beta}{1+\beta}\right)\right.\right. \\
& \left.\left.+\ln \left(\frac{1-\beta}{1+\beta}\right) \ln \left(\frac{(1+\beta)^{2}}{4 \beta}\right)-\frac{1}{2} \operatorname{Li}_{2}\left(\frac{(1-\beta)^{2}}{(1+\beta)^{2}}\right)+\frac{\zeta_{2}}{2}\right]\right\}
\end{aligned}
$$

and the result for diagram 2(e) is its negative: $I_{2 e}=-I_{2 d}$. The diagram of Fig. $2(\mathrm{f})$ gives

$$
I_{2 f}=\frac{1}{\epsilon}\left\{-\frac{1}{4}\left[2 \zeta_{2}+\ln ^{2}\left(\frac{1-\beta}{1+\beta}\right)\right]\left[\frac{\left(1+\beta^{2}\right)}{2 \beta} \ln \left(\frac{1-\beta}{1+\beta}\right)+1\right]+\frac{\left(1+\beta^{2}\right)}{12 \beta} \ln ^{3}\left(\frac{1-\beta}{1+\beta}\right)\right\} .
$$

Diagram 3(a) represents two graphs with overall $C_{F}^{2}$ color factor, $I_{3 a 1}=-3 /\left(2 \epsilon^{2}\right)+1 /(2 \epsilon)$, and another graph $I_{3 a 2}=1 / \epsilon^{2}-1 /(2 \epsilon)$. The quark-loop contribution to graph $3(\mathrm{~b})$ is $I_{3 b q}=$ $\left(n_{f} / 3\right)\left[1 / \epsilon^{2}-5 /(6 \epsilon)\right]$ while the gluon plus ghost loop contribution is $I_{3 b g}=(5 / 12)\left[1 / \epsilon^{2}-\right.$ $31 /(30 \epsilon)]$. Note the relation $d / d \beta\left[2 \beta I_{2 c} /\left(1+\beta^{2}\right)\right]=\left[-2 /\left(1-\beta^{2}\right)\right] I_{3 b}$. Finally diagram $3(\mathrm{c})$ gives $I_{3 c}=\left[\left(1+\beta^{2}\right) /(2 \beta)\right]\left(-1 / \epsilon^{2}\right) \ln [(1-\beta) /(1+\beta)]$.

Combining the above kinematic results with color and symmetry factors, the contribution of the diagrams in Figs. (2) and (3) to the two-loop soft anomalous dimension is

$$
\begin{aligned}
& C_{F}^{2}\left[I_{2 a}+I_{2 b}+2 I_{2 d}+2 I_{2 e}+I_{3 a 1}+I_{3 a 2}+I_{3 c}\right] \\
+ & C_{F} C_{A}\left[-\frac{1}{2} I_{2 b}+I_{2 f}-I_{2 c g}-I_{2 e}-I_{3 b g}-\frac{1}{2} I_{3 a 2}\right]+\frac{1}{2} C_{F}\left[I_{2 c q}+I_{3 b q}\right] \\
= & -\frac{1}{2 \epsilon^{2}}\left(\Gamma_{S}^{(1)}\right)^{2}+\frac{\beta_{0}}{4 \epsilon^{2}} \Gamma_{S}^{(1)}-\frac{1}{2 \epsilon} \Gamma_{S}^{(2)}
\end{aligned}
$$

where we have cancelled out factors of $\alpha_{s}^{2} / \pi^{2}$ on both sides of the equation. On the right-hand side of Eq. (5) in addition to $\Gamma_{S}^{(2)}$, which appears in the coefficient of the $1 / \epsilon$ pole, there also appear terms from the exponentiation of the one-loop result and the running of the coupling, with $\beta_{0}=(11 / 3) C_{A}-2 n_{f} / 3, C_{A}=3$, which account for all the double poles of the graphs. From Eq. (5) we solve for the two-loop soft anomalous dimension:

$$
\begin{aligned}
\Gamma_{S}^{(2)}= & \left\{\frac{K}{2}+\frac{C_{A}}{2}\left[-\frac{1}{3} \ln ^{2}\left(\frac{1-\beta}{1+\beta}\right)+\ln \left(\frac{1-\beta}{1+\beta}\right)-\zeta_{2}\right]\right. \\
& \left.+\frac{\left(1+\beta^{2}\right)}{4 \beta} C_{A}\left[\operatorname{Li}_{2}\left(\frac{(1-\beta)^{2}}{(1+\beta)^{2}}\right)+\frac{1}{3} \ln ^{2}\left(\frac{1-\beta}{1+\beta}\right)+\zeta_{2}\right]\right\} \Gamma_{S}^{(1)} \\
+C_{F} C_{A} & \left\{\frac{1}{2}+\frac{1}{2} \ln \left(\frac{1-\beta}{1+\beta}\right)+\frac{1}{3} \ln ^{2}\left(\frac{1-\beta}{1+\beta}\right)-\frac{\left(1+\beta^{2}\right)^{2}}{8 \beta^{2}}\left[-\operatorname{Li}_{3}\left(\frac{(1-\beta)^{2}}{(1+\beta)^{2}}\right)+\zeta_{3}\right]\right. \\
& \left.-\frac{\left(1+\beta^{2}\right)}{2 \beta}\left[\ln \left(\frac{1-\beta}{1+\beta}\right) \ln \left(\frac{(1+\beta)^{2}}{4 \beta}\right)-\frac{1}{6} \ln ^{2}\left(\frac{1-\beta}{1+\beta}\right)-\operatorname{Li}_{2}\left(\frac{(1-\beta)^{2}}{(1+\beta)^{2}}\right)\right]\right\} .
\end{aligned}
$$

We have written the two-loop result $\Gamma_{S}^{(2)}$ in Eq. (6) in the form of a term which is a multiple of the one-loop soft anomalous dimension $\Gamma_{S}^{(1)}$ plus additional terms. The well-known two-loop constant $K\left[11\right.$ is given by $K=C_{A}\left(67 / 18-\zeta_{2}\right)-5 n_{f} / 9$. Note that the color structure of $\Gamma_{S}^{(2)}$ involves only the factors $C_{F} C_{A}$ and $C_{F} n_{f}$; all $C_{F}^{2}$ terms cancel out. 


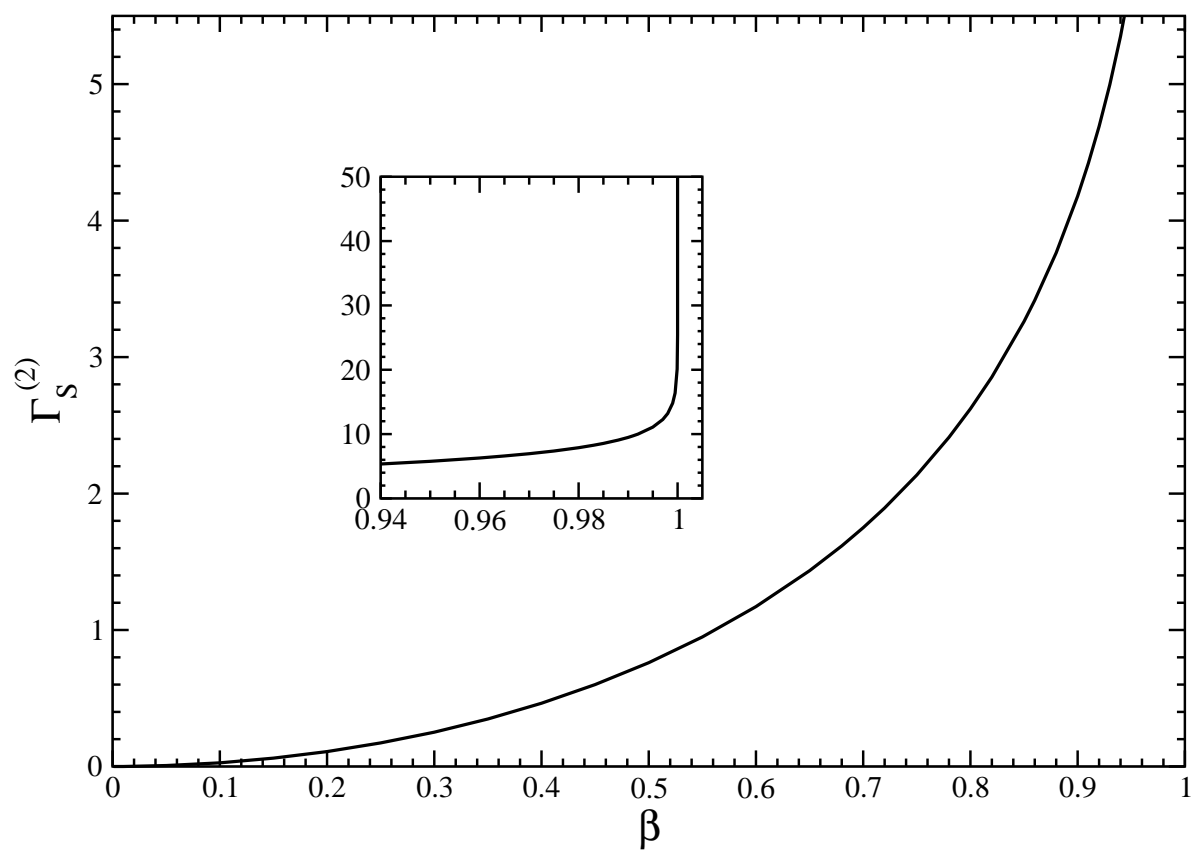

Figure 4: Two-loop soft anomalous dimension $\Gamma_{S}^{(2)}$ for top quark production via $e^{+} e^{-} \rightarrow t \bar{t}$.

The two-loop soft anomalous dimension for $e^{+} e^{-} \rightarrow t \bar{t}$ is plotted as a function of $\beta$ with $n_{f}=5$ in Fig. 4 . Note that $\Gamma_{S}^{(2)}$ vanishes at $\beta=0$, the threshold limit, and diverges at $\beta=1$, the massless limit. The inset in Fig. 4 shows more clearly the sharp increase of $\Gamma_{S}^{(2)}$ as $\beta$ approaches 1 . Figure 5 also displays $\Gamma_{S}^{(2)}$ but in a logarithmic plot which makes the small $\beta$ region more clear.

It is instructive to study the small and large $\beta$ behavior of $\Gamma_{S}^{(2)}$ in more detail. We first expand around $\beta=0$ and find

$$
\Gamma_{S \exp }^{(2)}=-\frac{2}{27} \beta^{2}\left[C_{F} C_{A}\left(18 \zeta_{2}-47\right)+5 C_{F} n_{f}\right]+\mathcal{O}\left(\beta^{4}\right)
$$

Note that $\Gamma_{S}^{(2)}$ is an even function of $\beta$ and hence only even powers of $\beta$ appear in the expansion. In Fig. 6 we see that just keeping the first term in the expansion as shown in Eq. (17) provides a good approximation to the complete result for small $\beta$. The ratio of the expansion to the complete $\Gamma_{S}^{(2)}$ remains within a few percent of unity for values of $\beta$ up to around 0.3 . Keeping more powers of $\beta$ in the expansion results of course in a better approximation. Keeping powers through $\beta^{12}$ results in an excellent approximation to $\Gamma_{S}^{(2)}$ over a wide range of $\beta$, starting to differ by a few percent above $\beta=0.8$ as seen from the corresponding curve in Fig. 6 .

As observed in Ref. [12], the two-loop soft anomalous dimension for processes with massless quarks is proportional to the one-loop result of Ref. [13] by the simple factor $K / 2$, i.e. $\Gamma_{S \text { massless }}^{(2)}=(K / 2) \Gamma_{S \text { massless }}^{(1)}$ This property was further explored and generalized in Refs. [14, 15, 16]. We see this $K / 2$ factor for heavy quarks as the first term in Eq. (6). It is clear that for the massive case the situation is rather more complicated than for massless processes: although all the $C_{F} n_{f}$ terms of $\Gamma_{S}^{(2)}$ are included in $(K / 2) \Gamma_{S}^{(1)}$, there are many additional $C_{F} C_{A}$ 


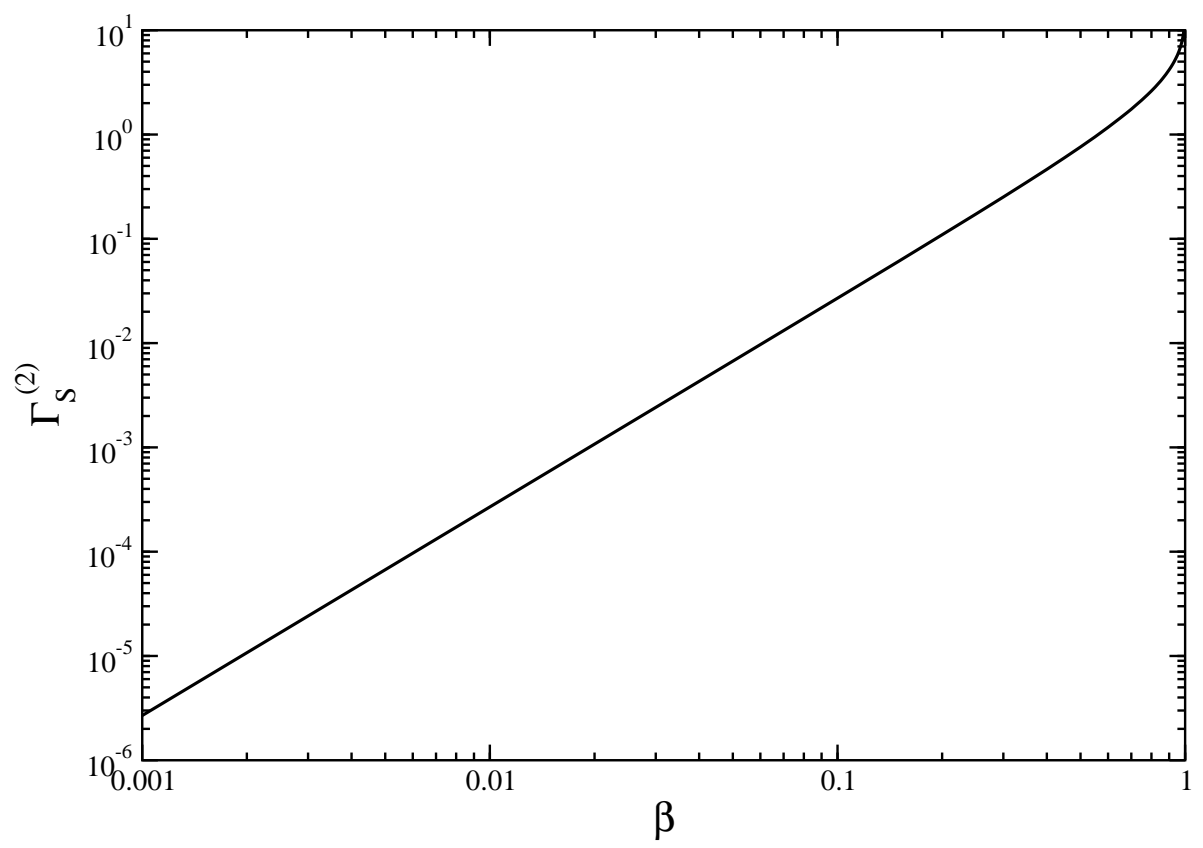

Figure 5: Logarithmic plot of $\Gamma_{S}^{(2)}$ for top quark production via $e^{+} e^{-} \rightarrow t \bar{t}$.

terms. It is interesting to check however that in the massless limit of our $\Gamma_{S}^{(2)}$ this relation is satisfied. In Fig. 6 we also plot the ratio of $(K / 2) \Gamma_{S}^{(1)}$ to $\Gamma_{S}^{(2)}$. We see that indeed the ratio is 1 at the limit $\beta=1$, i.e. the massless limit, so the massless proportionality relation is recovered. However, for any other value of $\beta$ the ratio is not one; in fact it is 1.144 near $\beta=0$, the threshold limit, gradually decreases with increasing $\beta$, and falls steeply to 1 in the region very close to $\beta=1$.

Given that the expansion around $\beta$, Eq. (7), gives very good approximations to $\Gamma_{S}^{(2)}$ at smaller $\beta$ while the expression $(K / 2) \Gamma_{S}^{(1)}$ is a better approximation at large $\beta$, we can derive an approximation to $\Gamma_{S}^{(2)}$ for all $\beta$ values by starting with the $\beta$ expansion of $\Gamma_{S}^{(2)}$, and then adding $(K / 2) \Gamma_{S}^{(1)}$ and subtracting from it its $\beta$ expansion:

$$
\begin{aligned}
\Gamma_{S \text { approx }}^{(2)} & =\Gamma_{S \exp }^{(2)}+\frac{K}{2} \Gamma_{S}^{(1)}-\frac{K}{2} \Gamma_{S \exp }^{(1)} \\
& =\frac{K}{2} \Gamma_{S}^{(1)}+C_{F} C_{A}\left(1-\frac{2}{3} \zeta_{2}\right) \beta^{2}+\mathcal{O}\left(\beta^{4}\right)
\end{aligned}
$$

As seen from Fig. 6, adding just the $\beta^{2}$ terms to $(K / 2) \Gamma_{S}^{(1)}$ as in Eq. (8) provides an excellent approximation to the exact result for $\Gamma_{S}^{(2)}$ for all $\beta$, never more than half a percent away from the exact value. It is quite remarkable that such a simple expression provides this good an approximation to a rather long and complicated expression for $\Gamma_{S}^{(2)}$. If we keep additional terms through order $\beta^{12}$ then the approximation is truly impressive, not differing by more than one per mille anywhere in the $\beta$ range, and in fact not differing by more than a few parts per million for most $\beta$. At a linear collider with $\sqrt{s}=500 \mathrm{GeV}, \beta=0.726$ for a top mass of 172 $\mathrm{GeV}$. For this value of $\beta$ the expansion through order $\beta^{12}$ of Eq. (7) as well as the approximate 


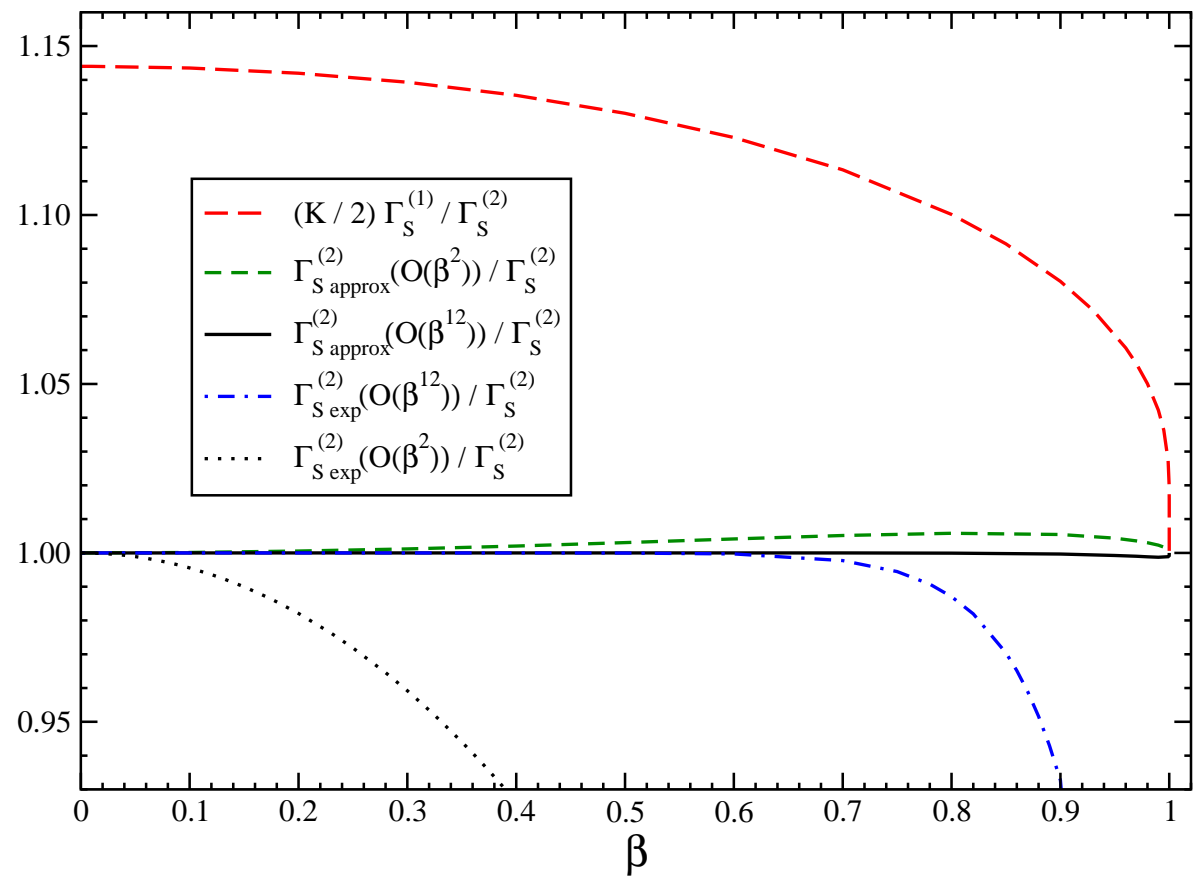

Figure 6: Expansions and approximations to $\Gamma_{S}^{(2)}$ for $e^{+} e^{-} \rightarrow t \bar{t}$.

expression at order $\beta^{2}$ or higher of Eq. (8) give excellent approximations. However, $(K / 2) \Gamma_{S}^{(1)}$ by itself is $11 \%$ larger than $\Gamma_{S}^{(2)}$.

The result in Eq. (6) can be written in terms of the cusp angle $\gamma=\ln [(1+\beta) /(1-\beta)]$ as

$$
\begin{aligned}
\Gamma_{S}^{(2)}=\frac{K}{2} \Gamma_{S}^{(1)}+C_{F} C_{A} & \left\{\frac{1}{2}+\frac{\zeta_{2}}{2}+\frac{\gamma^{2}}{2}-\frac{1}{2} \operatorname{coth}^{2} \gamma\left[\zeta_{3}-\zeta_{2} \gamma-\frac{\gamma^{3}}{3}-\gamma \operatorname{Li}_{2}\left(e^{-2 \gamma}\right)-\operatorname{Li}_{3}\left(e^{-2 \gamma}\right)\right]\right. \\
& \left.-\frac{1}{2} \operatorname{coth} \gamma\left[\zeta_{2}+\zeta_{2} \gamma+\gamma^{2}+\frac{\gamma^{3}}{3}+2 \gamma \ln \left(1-e^{-2 \gamma}\right)-\operatorname{Li}_{2}\left(e^{-2 \gamma}\right)\right]\right\},(9)
\end{aligned}
$$

where $\Gamma_{S}^{(1)}=C_{F}(\gamma \operatorname{coth} \gamma-1)$, and is consistent with the cusp anomalous dimension of Ref. [5].

Finally we consider the two-loop soft anomalous dimensions for single top and for top pair (or bottom or charm pair) production at hadron colliders. Since the two-loop soft anomalous dimensions for massless quarks obey the simple proportionality relation to one loop, and the diagrams for single top and top pair processes involve eikonal graphs with both massless and massive eikonal lines, it is clear that for $\beta$ near 1 the relation $\Gamma_{S}^{(2)}=(K / 2) \Gamma_{S}^{(1)}$ will hold for both single top and $t \bar{t}$ production. For smaller $\beta$, however, this relation is no longer valid. For reference, at the Tevatron and the LHC the $t \bar{t}$ cross section receives most contributions in the region around $0.3<\beta<0.8$ which peak roughly around $\beta \sim 0.6$. However, since even a $15 \%$ discrepancy between the exact $\Gamma_{S}^{(2)}$ and $(K / 2) \Gamma_{S}^{(1)}$ may result in less than $1 \%$ effect in the total cross section it may turn out that using the massless proportionality relation is adequate for many purposes (see also the related discussion in [17]). Nevertheless, a rigorous treatment requires that we include the extra terms for the massive case. More details will be given elsewhere. 
This work was supported by the National Science Foundation under Grant No. PHY 0555372 .

\section{References}

[1] W. Wagner, Rept. Prog. Phys. 68, 2409 (2005) hep-ph/0507207;

A. Quadt, Eur. Phys. J. C 48, 835 (2006);

R. Kehoe, M. Narain, and A. Kumar, Int. J. Mod. Phys. A 23, 353 (2008), arXiv:0712.2733 [hep-ex];

T. Han, Int. J. Mod. Phys. A 23, 4107 (2008), arXiv:0804.3178 [hep-ph];

W. Bernreuther, J. Phys. G. 35, 083001 (2008), arXiv:0805.1333 [hep-ph];

D. Wackeroth, in ICHEP 2008, arXiv:0810.4176 [hep-ph].

[2] CDF Collaboration, F. Abe et al., Phys. Rev. Lett. 74, 2626 (1995) hep-ex/9503002];

D0 Collaboration, S. Abachi et al., Phys. Rev. Lett. 74, 2632 (1995) [hep-ex/9503003].

[3] D0 Collaboration, V.M. Abazov et al., arXiv:0903.0850 [hep-ex];

CDF Collaboration, T. Aaltonen et al., arXiv:0903.0885 [hep-ex].

[4] N. Kidonakis and G. Sterman, Phys. Lett. B 387, 867 (1996);

Nucl. Phys. B505, 321 (1997) [hep-ph/9705234.

[5] S.V. Ivanov, G.P. Korchemsky, and A.V. Radyushkin, Yad. Fiz. 44, 230 (1986) [Sov. J. Nucl. Phys. 44, 145 (1986)];

G.P. Korchemsky and A.V. Radyushkin, Phys. Lett. B 171, 459 (1986);

Nucl. Phys. B283, 342 (1987); Phys. Lett. B 279, 359 (1992) hep-ph/9203222.

[6] N. Kidonakis, Phys. Rev. D 74, 114012 (2006) hep-ph/0609287.

[7] E. Eichten and R. Jackiw, Phys. Rev. D 4, 439 (1971);

A. Sen, Phys. Rev. D 24, 3281 (1981);

S. Aoyama, Nucl. Phys. B194, 513 (1982);

J.G.M. Gatheral, Phys. Lett. 133B, 90 (1983);

J. Frenkel and J.C. Taylor, Nucl. Phys. B246, 231 (1984);

D. Knauss and K. Scharnhorst, Annalen Phys. 41, 331 (1984);

A. Bassetto, I.A. Korchemskaya, G.P. Korchemsky, and G. Nardelli, Nucl. Phys. B408, 62 (1993) hep-ph/9303314;

I.A. Korchemskaya and G.P. Korchemsky, Nucl. Phys. B437, 127 (1995) hep-ph/9409446];

C.F. Berger, Phys. Rev. D 66, 116002 (2002) [hep-ph/0209107].

[8] N. Kidonakis, hep-ph/0208056; in DIS 2003 (PNPI, Gatchina, 2003), p. 541 hep-ph/0307145].

[9] N. Kidonakis, A. Sabio Vera, and P, Stephens, arXiv:0802.4240 [hep-ph];

N. Kidonakis and P. Stephens, in DIS 2008, arXiv:0805.1193 [hep-ph].

[10] N. Kidonakis, in PANIC 2008, arXiv:0901.2155 [hep-ph]. 
[11] J. Kodaira and L. Trentadue, Phys. Lett. 112B, 66 (1982).

[12] S.M. Aybat, L.J. Dixon, and G. Sterman, Phys. Rev. Lett. 97, 072001 (2006) [hep-ph/0606254]; Phys. Rev. D 74, 074004 (2006) hep-ph/0607309].

[13] N. Kidonakis, G. Oderda, and G. Sterman, Nucl. Phys. B531, 365 (1998) hep-ph/9803241.

[14] T. Becher and M. Neubert, arXiv:0901.0722 [hep-ph]; arXiv:0903.1126 [hep-ph].

[15] E. Gardi and L. Magnea, JHEP 03, 079 (2009), arXiv:0901.1091 [hep-ph].

[16] L.J. Dixon, arXiv:0901.3414 [hep-ph].

[17] N. Kidonakis and R. Vogt, Phys. Rev. D 68, 114014 (2003) hep-ph/0308222];

Phys. Rev. D 78, 074005 (2008), arXiv:0805.3844 [hep-ph]. 